since these are frequent in the region to the southeast of Fiji. In the now Suva instrument and other Milne-Shaw seismographs of recent date, Mr. J. J. Shaw has made an improvement by having the agate cups surrounded by light aluminium bands so that the seismograph is not jerked out of action by severe local shocks.

Dr. A. E. MI. Geddes has been making observations with the Milne-Shaw instruments at Aberdeen, using the method developed by Dr. A. W. Lee at Kew, to see whether the free motion of the seismograph was in accordance with the mathematical theory of dumped periodic motion, and to find whether or not the period could be determined by removing the damping magnets. Ho found that the agreement with the theory was very satisfactory, but that an increase of the order of $10^{-1}$ sec. in the period may havo been due to damping. Dr. Geddes suggests that this may havo been on account of the boom of the pendulum being somewhat paramagnetic.

The Jagger shock recorder built for the Association at Bristol under the supervision of Dr. C: F. Powell has been installed at Dunira, Comrie, Perthshire, in a croquet house, through the courtesy of Mr. W. G. Macbeth. Its position is lat. $56^{\circ} 23^{\prime} 19^{\prime \prime} \mathrm{N}$., long. $4^{\circ} 2^{\prime} 41^{\prime \prime}$ W., three miles north northwest of the Highland boundary fault. The pendulum oscillates in a vertical plane orientated N. $76^{\circ} \mathrm{E}$., is adjusted to a period of nearly a second, and is damped by means of a metal fin dipping into an oil bath. It is intended to record only local shocks; the records aro obtained on smolied disks, which are changed every twenty-four hours. An anonymous donor has offered to provido for somo years an honorarium to tho keeper, and this has been gratefully accepted by the com. mittee. During the year the chief local shocks recorded at Comrie have been October. 15, and November 12, 1938, January 23, May 26, 31, and June 11, 1939. None of these was recorded by other seismographs in Great Britain or felt by people. They may be supposed to have been due to movements at the boundary fault. British tremors probably duo to subsidences have been reported to
Dr. Dollar as having occurred at Brierly, Yorkshire, on November 12, 1938, and at Porth, Glamorganshire, on December 18, 1938. From October 1939 Dr. Dollar will bo pleased to receive observations of British earthquakes at the Geology Department, University of Glasgow.

At Oxford the work of the International Seismological Summary has continued, and through the courtesy of the American Museum of Natural History in New York, two Mollweide projection maps centred on $160^{\circ}$ longitude have been prepared, one for deep focus epicentres and the other for all epicentres used in the I.S.S. during the years 1913-1933. Copies of these maps were sent to Washington for the meeting of the International Union of Geodesy and Geophysics held last month, and it is noteworthy that at this meeting a seismological committee under the chairmanship of Dr. S. W. Visser was to discuss technical details concerning the I.S.S., including the possible use of geocentric co-ordinates instead of geographic co-ordinates. Dr. H. Jeffreys, who has been appointed a member of this international committeo, has been active recently in computation, chiefly from the data of deep-focus earthquakes, of the travel times of various phases of earthquake waves, including those reflected and refracted at the core. For this Iatter purpose the radius of the outer core had to be evaluated from the available data, and this is now estimated to be $3473 \pm 4 \mathrm{~km}$. Dr. R. Stoneley has continued his study of Rayleigh waves and Love waves, and now belioves that significant differences in the relative frequency of these in different earthquakes imply differences in the types of initial movement at the foci. One of the pioneers of British seismology was the late Dr. John Milne, who wrote the book "Earthquakes and other Earth Movements". As a result of the progress made, often as the result of work initiated by Milne himself, Dr. A. W. Lee has practically had to rewrite this book though keeping to the form devised by Milne. The revised book has been published during the year under the title "Earthquakes and other Earth Movements" by J. Milne and A. W. Lee (see NATURE, 143, $872 ; 1939)$.

\title{
THE ETHYL-THIOGLUCOSIDES AND A NEW MONOACETONEGLUCOSE
}

N attempting to synthesize disaccharides from
glucosediethylmercaptal, Brigl, Gronemeier and
Schultz (Ber. deutsch. chem. Gesell., Nay 1939) have
obtained an. ethyl-thioglucoside with properties very
different from those of the $\alpha$ - and $\beta$-forms described
by Schneider and Sepp (Ber., 1916 and 1918). It is
now maintained that the new strongly dextro-
rotatory compound and the lievo-rotatory so-called
$\beta$-form both possess the pyranose ring structure,
whilst the dextro-rotatory $\alpha$-form must be a furano-
side. Convincing proof of the structure of this
furanoside is obtained from experiments with acetone
derivatives in which the 5.6 positions must be
occupied by the iso-propylidene group. By con-
densation of glucose diethylmercaptan with acetone
in presence of a finely powdered preparation of copper
sulphate from which just half the normal water of
crystallization had been removed, 5.6-monoiso-
propylidene mercaptal was prepared, from which, with the aid of mercuric chloride and cadmium carbonate, 5.6-acetonethioglucoside was formed.

Any doubt as to the positions occupied by the isopropylidene group is removed by the elimination in one stage of the two mercaptan groups of the mercaptal with formation of $\mathfrak{a}$ hitherto unknown 5.6-monoacetoneglucose, which reacts with more acetone to give the well-known 1.2.5.6-diacetone derivative. Thus Schneider and Sepp's $\alpha$-compound must be a furanoside and tho now monoacetone. glucose a glucofuranose, since the possibility of forming a pyranose ring is definitely oxcluded.

An interesting reaction occurs between this gluco. furanose and Schiff's reagent. The colour is restored slowly but clearly within a few minutes and fades again after long standing. This is explained by the tendency of the furanose to revert first to the aldehydic open-chain structure and later, after fission of the acetone group in the presence of acid, 
to the more stable pyranose ring. The $\beta$-pyranose structure of Schneider and Sepp's $\beta$-compound is inferred from its synthesis from acetobrom. glucuse by interchange of halogen for the mercaptan group.

No definite proof of the structure of the new ethylthioglucoside is given, but its stability to acid leads the authors to infer that it must be the corre. sponding $\alpha$-pyranoside. It was prepared by condensing glucose-diethylmercaptal with glucose in acid solution and was purified by conversion to its totracetate, which was isolated in a pure state and hydrolysed by baryta to the $\alpha$-thioglucosepyranoside. A remarkablo property is its high specific rotation, $+268.8^{\circ}$, which is about $100^{\circ}$ higher than the value calculated from Hudson's rule.

\section{ELECTRICAL EQUIPMENT OF BUILDINGS}

$\mathrm{T}$ HE new (eleventh) edition of the regulations of the Institution of Electrical Engineers for the wiring of buildings was published in June (London : Spon. Cloth 1s. 6d. net; paper cover 1s. Also from the Institution of Electrical Engineers).

These regulations enumerate tho main requirements and precautions considered necessary for ensuring satisfactory results, including safoty from fire and shock, in connoxion with the distribution of electrical energy in and about all types of dwelling houses, business premises, public buildings and factories, whether tho electric supply is derived from an external source or from private generating plant. With a public supply they are only applicable to the consumer's side of the consumer's terminals. In no circumstances are they applicable to telephono circuits other than radio circuits; and they only apply to radio circuits where such circuits are connected directly or indirectly to a public or private supply system. Primarily the regulations are intended to apply to low-voltage installations, but the cases of electrode water heaters, luminous discharge tubes and electric signs are mentioned. They are not intended to take the place of a detailed specification or to instruct untrained persons. Whenever applic. able they are supplementary to statutory regulations, such as those issued by the Electricity Cornmissioners and by the Home Office, and they include also the requirements for theatres and for safety in mines.

Various methods of carrying out the electrical equipment of buildings are considerod and in order to guard against the risk of fire and shock tho method selected must be suitable for the voltage, the atmospheric conditions, the size of the installation and the type of building. There is no intention of discouraging invention or of excluding other materials and methods which may bo approved in the futuro.

The Committee of the Wiring Rules is fully repre. sentativo of all those connected with the industry. It has representatives from the Association of Con. sulting Engineers, the B.E.A.M.A., the Cable Makers Association, the Fire Offices Committee, the Home Office, the Electrical Contractors Association and others. The Committee holds meetings periodically and all difficulties are fully discussed and reported on. The revision has been carried out very thoroughly, but contractors whose work has hitherto been of the highest quality will not be affected.

\section{REINFORCED CONCRETE COLUMNS}

\begin{abstract}
UNDER the usual applications of column theory the theoretical values for loads at failure are dependent on the elasticity of the material of which the column is made, and it is assumed in this theory that the material is both homogeneous and isotropic. In the case of reinforced concrete this assumption cannot be made, so that the applicability of the usual theories is not justifiable without experimental evidence of the actual failing loads. In particular, the effect of inelastic deformations of the concrete requires to be investigated.

An investigation, undertaken to determine the strength of long columns in short-period loading tests to destruction for cases where the initial eccentricity of loading is so small as to bo capable of being regarded as 'accidental' in practice, has been carried out at the Building Research Station, Garston, in co-operation with the Reinforced Concrete Associa. tion and under the supervision of Dr. W. H. Glanville. A report has been published by the Department of Scientific Research by the issue of a Building Rosearch Technical Paper (No. 24. H.M. Stationery Office. $9 d$. net), which is the seventh in a series of Studies in Reinforced Concrete.
\end{abstract}

Even for well-defined initial conditions of loading it is impossible to carry out a rigorous mathematical analysis of the stress distribution which, by reason of the effects of ereep, must be in some way a function of the time the material is under load. The experiments havo shown, however, that a simple approximate analysis can be used to estimate the strength of long columns under the conditions spocified. The paper opens by showing how an expression may be derived for the valuo of the ratio of the applied load to the short column load in terms of the Euler load, the short column load and a coefficient proportional to the slenderness ratio. The short-period tests to destruction are analysed and cited to show this formula to be satisfactory for particular values of this coefficient.

The tests were made on columns 6 in. square in section, but in some cases, whore high values of the slenderness ratio were required, a 3 -in. square section was used. The columns and the arrangements for testing are illustrated by photographs and the test results are set out in tables and diagrams in which are shown also comparisons between the theoretical and the practical figures. 